\title{
1 An associative account of avian navigation
}

2

3

$4 \quad$ Tim Guilford \& Theresa Burt de Perera

5 Animal Behaviour Research Group,

6 Department of Zoology

$7 \quad$ South Parks Road

8 Oxford

$9 \quad$ UK

10

11 Tim.guilford@zoo.ox.ac.uk

12

13

Theresa.burt@zoo.ox.ac.uk

14 
An associative account of avian navigation

\section{Navigational concepts and mechanisms}

Though many puzzles remain (e.g. Alerstam, 2006), the study of long-distance orientation in birds has produced some astonishing biological discoveries, such as innate vector control of migration (see Alerstam, 1990) or the magnetic sense in animals (see Wiltschko \& Wiltschko, 1995), and provided an enduring

23 framework for understanding animal movement behaviour through the

24 development of various navigational map and compass concepts (for a summary 25 see Wiltschko \& Wiltschko, 2009). The conceptual approach to avian navigation 26 has generally if implicitly been to attempt to understand the sensory-

27 informational basis of these systems as if they were dedicated modules 28 adaptively designed to solve the navigational challenges that birds are thought to 29 face. But often discussion of these concepts has been silent on the issue of the 30 underlying learning mechanisms required to inform their effectiveness. Instead

31 we take a bottom-up approach, and try to outline the extent to which

32 navigational phenomena might be understood in terms of simple associative

33 learning mechanisms. We argue that whilst most concepts fit readily with what is

34 known about animal learning, some may need rethinking because they appear to 35 require significant departure from these known principles. We explore one issue 36 in detail, the apparent cognitive complexities required by extrapolated 37 navigational map concepts such as the gradient (or grid) map, suggesting a 38 potential refocus of attempts to understand the olfactory map in particular. 
40 Since much is now understood about how animals learn about the contingencies

41 in their environment through associative (and related) learning mechanisms

42 (e.g. Pearce, 2008), enabling them to operate adaptively in a wide variety of tasks

43 and contexts, we take the stance that we should expect the same mechanisms to

44 underlie animal navigation too. What we mean by 'associative mechanisms', at

45 the risk of caricaturing a huge and sophisticated body of knowledge and debate,

46 is the set of fundamental processes that enable the stable neural representation

47 by animals of the contingencies between perceived stimuli, events or actions, and

48 the apparent hedonic consequences of these. Put simply, actions become guided

49 (and here we mean significant movements) by the formation of links between

50 perceived stimuli, responses, and outcomes, that result from an animal's direct

51 experience of the world. We contrast this with the idea that animals may employ

52 specific modules designed to be filled with specialized information to allow them

53 to operate adaptively in situations far outside of their direct experience. We

54 start by emphasizing that some phenomena in avian navigation (broadly

55 defined) do indeed owe their control to non-associative, highly specialized

56 mechanisms. For many first-time migration tasks, for example, especially those

57 performed by short-lived taxa, an innate toolbox for orienting may be needed

58 because the information required to make movements adaptive cannot be learnt

59 in time to be useful, and there is good evidence that birds in particular are

60 equipped with such a toolbox (e.g. Alerstam, 1990). So, associative mechanisms

61 cannot account for everything that controls long-distance movement in birds.

62 However, for much of what is considered to be navigation, the mechanisms of

63 control may more simply be the result of associative learning of the

64 contingencies between salient environmental cues and outcomes of importance 
to the animal (such as getting out to and back home from the location of a valuable resource, using the appearance or direction of a distant landmark close to that location).

In this essay we develop briefly the idea that avian navigation might be understood using a simple associative perspective. The intention is not to diminish the impressive discoveries that have been made in the field over many decades, instead it is to suggest that an associative perspective may help us decide where we do and where we do not need distinct and separate conceptual frameworks to account for these discoveries. At least, our approach may provide a null-hypothesis for studies of navigation based on known mechanisms of learning; a baseline against which the special abilities of birds, or lack thereof, may be judged objectively.

\section{An associative account}

Our premise is that as a consequence of needing to explore space to find resources, and to return home (or to other places) efficiently, a bird will learn to navigate using simple associative processes. As the bird moves about its environment, it learns that certain stimuli or actions are associated with finding resources or returning home (are rewarding) and that others may signal or lead to delays or dangers (are punishing). These could be sequences of landmarks, or they could be the following of directional cues associated with places. That could lead simply to route following using the available visual landmarks (Guilford \& Biro, 2014), and it could lead to use of solar cues or magnetic cues to get home 
from certain directions. See Figure 1 for simple schematic. The improvement of

91 route efficiency may result as a consequence of slight deviations from a learnt

92 route being rewarded by faster homing. Consistent reward for homing from a

93 particular stable direction could lead to directional cues (compasses) controlling

94 orientation even at very familiar sites (Fuller et al., 1983). In the extreme, as

95 with the artefactual case of trained racing pigeons, directional control may come

96 to dominate orientation from any location if the rewarding direction becomes

97 the sole best predictor of homing.

99 The first expectation of our associative perspective is that birds will learn to 100 navigate using whatever salient environmental cues are available. Because 101 visual landscape cues are likely to be highly salient to such visual animals as 102 birds, and because they are also often geographically stable and distinct, we may 103 expect these to become important in guiding learnt goal orientation. Over larger 104 distances, however, or where approximate directions provide the most efficient 105 balance between information storage and navigational performance, more 106 general cues from the environment might be more salient: cues such as olfactory 107 gradients or parameters of the earth's magnetic field. Where the availability or 108 suitability of different cue types varies then different navigational systems might 109 be learnt by different individuals, or in different locations or environments.

110 Indeed, if this variation is itself predictable, depending for example on weather

111 conditions, an associative perspective might also accommodate individuals 112 learning multiple navigational cues and the ability to switch between them in a 113 context dependent way. Both these possibilities might give the appearance of 114 cue redundancy or a hierarchical navigational system (e.g. Wiltschko \& 
115 Wiltschko, 2009), as if these were somehow additional adaptive properties of a 116 specialized navigational capacity in birds.

118 Thus, our associative approach can in principle account for the development of 119 many commonly noted navigational behaviours, such as route-following 120 behaviour, where birds pilot between known places features using the 121 arrangement of landmark features (Guilford \& Biro, 2014), or compass122 dependent navigation where birds learn to associate homeward compass 123 directions with places they have visited before (see Figure 1). Indeed, even the 124 sun-compass itself might be seen as the product of an associative process 125 connecting solar azimuthal position with temporal context through experience 126 (Guilford and Taylor, 2014).

\section{Descriptive versus extrapolated maps}

130 So far, our proposal is that animals acquire maps or other spatially informative 131 representations (e.g. compasses) that are descriptive of the environment 132 through which they move, reinforced by actual contingencies encountered as a 133 consequence of those movements. This seems uncontroversial. We have said 134 little that cannot be found at least implicitly in previous theoretical treatments, 135 such as that of Baker (1984). If we think about navigational abilities from a 136 mechanistic learning perspective we find that most traditional concepts can be 137 given a simple associative account. But not all. Some navigational concepts 138 suppose that animals use maps that allow them to extrapolate their position to 139 completely unfamiliar places, not just to describe their encountered 
environment. And it is this distinction that concerns us for the rest of this essay

141 because we think it potentially has profound implications for spatial learning in

142 animals.

144 The most obvious of these extrapolated maps is the so-called gradient map, or

145 grid map (for terms and concepts see Able, 2001), because it is hypothesized to

146 consist of a representation of large scale (non-parallel) environmental gradients

147 allowing position fixing from places never visited before (and without the

148 integration of outward journey information). Many possible environmental cues

149 have been investigated or considered, including the features of the sun's arc,

150 gradients in magnetic intensity and dip angle, the Coriolis force and gravity (see

151 Baker, 1984; Wiltschko \& Wiltschko, 2009). Whilst past researchers have mainly

152 concerned themselves with the nature of the cues themselves, our point here is

153 that whatever the cues involved such a map is premised on the idea of

154 extrapolation. It must involve the learning of environmental relationships to

155 build a map whose adaptive purpose is to facilitate navigation from places not

156 yet visited (and therefore in the absence of directly rewarding feedback). The

157 potential power of a gradient map is precisely that it can be extrapolated far

158 beyond the stimulus values perceived during its acquisition, but it is exactly this

159 extrapolation that makes the idea problematic for a mechanistically simple

160 associative account. At least some versions of the olfactory map hypothesis also

161 have this extrapolation property (whether or not we choose to call them gradient

162 maps). For example, Gagliardo (2013) provides a clear description of the

163 olfactory hypothesis as it relates to studies of pigeon navigation especially. She

164 makes explicit that the learning of relationships (at or around the home colony) 
165 between odour signatures and geographic direction (deduced either directly

166 from ranging in the familiar area or indirectly from the directions of the winds

167 on which odour types arrive) functions to facilitate homeward orientation from

168 completely unfamiliar places by interpretation of the up-gradient olfactory

169 character of the novel site. At least in this version, the learnt olfactory map is

170 designed to be extrapolated.

171

172 As was perhaps realized by Baker when he suggested that grid (gradient) maps,

173 if they existed, would alone raise the phenomenon of bird navigation from the

174 mundane to the mysterious (Baker, 1984), any extrapolated map provides a

175 fundamental mechanistic challenge. This challenge is quite separate from that of

176 determining which environmental factors are involved in the map - the usual

177 concern of students of navigation. The challenge is to understand whether it is

178 really the case that birds (say) are learning the details of their navigational map

179 without directly rewarding feedback from the successful application of that map.

180 Put simply, are navigators really capable of acquiring a map of unfamiliar places

181 by learning its characteristics over familiar terrain? This is not impossible of

182 course, but it is intriguing, and intriguing in a mechanistic way that was perhaps

183 not recognized by the original proponents of such maps. It would, we argue,

184 require the presence of a rather specific cognitive module primed to acquire

185 memories about environmental contingencies that are inferred or predicted

186 rather than actually experienced. Indeed, Jacobs (2012) has recently argued for

187 just such a system driving the evolution of cognitive structures in general, and

188 originating in the need for olfactory spatial learning which could extract and 
represent bearing-related spatial information in both a descriptive and predictive way (i.e. at least partially involving extrapolation).

What, then, is the evidence that navigating animals use extrapolated maps, and therefore require mechanisms beyond simple associative learning for navigation? In his 1984 review, Baker concluded that the evidence for grid maps in birds was equivocal. 20 years later, Wallraff (2005) largely concurs with this view in his detailed review. On the other hand, evidence in support of some form of olfactory map in birds has accumulated steadily over the past 40 years (Papi, 1992; Gagliardo, 2013), even though challenges continue to be raised (e.g. Jorge et al., 2010) and countered (Gagliardo et al., 2011). Again, our aim here is not to review or take a position on the precise sensory nature of the navigational map in birds (where it has been best studied), but to ask whether there is robust evidence yet that it relies on extrapolation.

This question may at first seem puzzling, because it is almost axiomatic of navigational displacement experiments that subjects are transported to completely unfamiliar places and released, after some experimental manipulation of interest and often in the absence of outward journey information that might allow computation of the home direction through some form of path integration. Scores of experiments have shown that pigeons especially are capable of homeward orientation after such displacements, at least as long as their olfactory sense is intact (Gagliardo, 2013; Wallraff, 2005). Whilst such experiments do support the idea that birds have an olfactory map, we need to scrutinize more carefully whether they prove that the map is extrapolated in 
214 light of the fact that this distinction has become mechanistically crucial. We do

215 not think that the evidence for a genuinely extrapolated map is yet clear.

\section{Homeward orientation at unfamiliar sites}

The strongest claim for our proposal would be that the apparent ability to orient

220 homeward from an unfamiliar location in displacement experiments may also

221 arise as a product of generalization from an ability to navigate successfully in the

222 familiar area, rather than providing unequivocal evidence for an extrapolated

223 navigational map. To clarify this idea, we consider how it might work in the case

224 of olfactory navigation (although in principle it applies with equal force to any

225 navigational map that supposedly works by extrapolating from environmental

226 gradients experienced by the navigator, magnetic gradients included).

228 Suppose that birds exploring their environment register that odour

229 concentrations or ratios vary in different directions, so that olfactory gradients

230 become learnt as distinctive of directional relationships within that expanding

231 familiar environment. Thus, an olfactory map of the familiar environment

232 emerges as a result of direct contact with it, and perhaps the directionally

233 variable winds over it, during exploration. This learnt map then facilitates better

234 goal orientation and more efficient movement within that familiar area. Perhaps

235 olfactory cues lend themselves to such mapping because of their graded nature

236 and spatial scale, with visual landmarks being learnt later or for more precise

237 navigation because of their more local nature. So whilst it seems reasonable to

238 suppose that relationships and contingencies involving olfactory cues may be 
acquired for homeward guidance in the familiar area, in very few experiments

240 has the efficacy of olfactory-based navigation been explicitly tested at familiar

241 sites, although in those few the evidence shows that olfaction does indeed

242 improve homeward orientation (e.g. Gagliardo et al., 2001b).

244 But why should olfactory navigation generate apparently sensible orientation

245 from unfamiliar sites if, as we are here forced to argue, this is not its purpose?

246 The argument (which we caricature in several forms in Figure 2) would be that a

247 bird displaced artificially to an unfamiliar distant site is equipped only with

248 memories of associations between cues and orientation in its familiar area, but

249 that the bird may generalize to one or more of these if the stored representations

250 of environmental cues are similar to those currently perceived at the unfamiliar

251 site. In the absence of alternative sensible movement instructions, the animal

252 may respond to that generalization (Case 2 in Figure 2). Generally we might

253 expect that response to be weak. However, in some circumstances

254 discrimination learning can in fact produce stronger than normal responses

255 through a process known as peak shift (e.g. Weary et al., 1993), where a difficult

256 discrimination between negative and positive stimuli on a dimension generates

257 exaggerated response to stimuli displaced beyond the positive stimulus along the

258 dimension away from the negative. If we imagine, then, that a bird has learnt in

259 its familiar area to fly west to home when experiencing a particular odour ratio

260 that is only apparently slightly different from that experienced at home, then

261 experiencing a ratio that exaggerates the difference between these two might

262 lead to a strong response in the correct direction to home (Case 4 in Figure 2). 
264 This argument and our hypothetical odour ratio example appears to look more

265 like a mechanism by which extrapolated navigation could be achieved through

266 learning in the familiar area than an alternative account. So it is important to be

267 clear about the differences. If sensible orientation appears at unfamiliar sites

268 because the animal is accessing an extrapolatory navigation mechanism, then we

269 would expect orientation and homing at such sites to be good because this is

270 what must have provided the fitness feedback promoting the mechanism. On the

271 other hand, if it is an artefact of familiar area orientation and without real

272 ecological validity, then we might expect orientation and homing to be poor.

273 Even if peak responding were to shift up the stimulus gradient away from values

274 already experienced, it will not do so indefinitely so at some distance we would

275 expect responding to become weak. These are perhaps not easy things to test,

276 but crucially it would be reasonable to expect a mechanism for navigation from

277 unfamiliar places to operate more effectively at unfamiliar sites than familiar

278 ones. Since researchers have not generally considered that olfactory navigation,

279 for example, might actually be a familiar area mechanism rather than an

280 extrapolated navigational one, these kinds of data are not directly available.

281 Indeed, the apparent efficacy of visual landscape cues in controlling orientation

282 once places are familiar (for a review see Guilford \& Biro, 2014) means that

283 olfactorily-controlled orientation would probably be overshadowed in such

284 releases, and the investigation of the relative efficacy of an olfactory association

285 in familiar versus unfamiliar places would require special experimental

286 considerations. 
Nevertheless, some experiments do confirm that olfactory cues can exercise

289

290

291

292

293

294

295 translates so poorly into actual homing. 
313 An obvious objection to our alternative interpretation of apparent olfactory

314 navigation at unfamiliar release sites, and favouring the hypothesis that it may

315 rely on some form of special learning module, is that fact that young pigeons

316 appear able to learn to use olfactory cues from distant sites on first release even

317 when they have been bred confined in an aviary as long as they are allowed

318 access to environmental air from different wind directions (e.g. Gagliardo et al.,

319 2001a). They appear to have learnt inside the aviary what they will need to

320 navigate outside it. Indeed, this ability appears to show an early sensitive period.

321 Thus, if birds have simply learnt contingent relationships between odours and

322 wind directions inside their aviary, then our associative account would require

323 that they are literally orienting around their aviary using such information. We

324 accept that this may seem implausible, but suggest that the possibility that

325 directional odours may be used in combination with wind direction in very short

326 range orientation might be investigated further. It should also be said that even

327 though pigeons respond sensibly to odour directions apparently learnt confined

328 within an aviary, navigational performance is generally improved in birds that

329 are allowed to range freely around their loft, suggesting that much more

330 information may be acquired when birds are indeed able to use that information

331 actively. But again, experiments relating the scale of exploratory movements

332 explicitly with the efficacy of the acquired map have not yet been done.

334 In short, given that navigation experiments have generally been designed to

335 investigate the sensory nature of navigational maps rather than their

336 representational or associative structure, these experiments do not robustly

337 inform us as to whether the extrapolated navigational abilities reported are core 
to the nature of the map (and hence indicative of a specialized spatial learning

339 module of some sort), or peripheral artefacts of experimental design and

340 consistent with a simple and general associative mechanism.

342 A null hypothesis for navigation

344 We argue that much of what we know about bird navigation may be founded in 345 simple underlying associative mechanisms. However, thinking about navigation 346 from an associative perspective has also clarified that some concepts commonly 347 referred to in the navigation literature would require the existence of more 348 complex underlying mechanisms, re-focussing the need for scrutiny of their 349 empirical basis. In particular, we consider that maps requiring extrapolation of 350 learnt spatial relationships rather than just descriptions of them, appear to

351 conflict with this associative perspective. Gradient (or grid) maps, and some 352 forms of olfactory map, appear to have this extrapolated property. Since there is 353 now considerable empirical evidence in support of an avian olfactory map the 354 question of the extent to which it genuinely functions as an extrapolated map 355 adapted for navigation from unfamiliar places when birds have no access to 356 familiar cues or outward journey information becomes critical. Here we have 357 suggested ways in which a key outcome of navigational displacement 358 experiments, homeward orientation from unfamiliar sites, could be generated 359 artefactually by generalization from associations learnt for familiar area 360 navigation. This emphasizes, for example, the need to study the relative 361 functionality of olfactory navigation within and beyond the bird's familiar area. 
Thus, we hope that our associative account may at least act as a principled null-

363 hypothesis for future studies of avian navigation.

\section{Acknowledgments}

We thank all in Oxnav for thought-provoking discussion, and the referees and editors of Journal of Avian Biology for insightful comments..

\section{References}

Able, K. P. 2001. The concepts and terminology of bird navigation. J. Avian Biol.,

Alerstam, T. 1990. Bird migration. Cambridge Univ. Press, Cambridge.

Alerstam, T. 2006. Conflicting evidence about long-distance animal navigation.

Science 313: 791-794.

Baker, R. R. 1984. Bird navigation: The solution of a mystery? London, U.K.:

Hodder \& Stoughton.

Fuller, E., Kowalski, U., and Wiltschko, R. 1983. Orientation in homing pigeons: compass orientation vs piloting by landmarks. J. Comp. Physiol. A, 153: 55-58.

381 Gagliardo, A. 2013. Forty years of olfactory navigation in birds. J. Exp. Biol. 216: 2165-2171.

383 Gagliardo, A., Ioalè, P., Odetti, F., and Bingman, V. P. 2001a. The ontogeny of the 384 homing pigeon navigational map: evidence for a sensitive learning period. Proc. 385 Roy. Soc. B, 268: 197-202.

386 Gagliardo, A., Odetti, F. and Ioalè, P. 2001b. Relevance of visual cues for 
orientation at familiar sites by homing pigeons: an experiment in a circular arena. Proc. Roy. Soc. B, 268: 2065-2070.

389 Gagliardo, A., Ioalè, P., Filannino, C. and Wikelski, M. 2011. Homing pigeons only

390 navigate in air with intact environmental odours: a test of the olfactory

391 activation hypothesis with GPS data loggers. PLoS ONE 6: e22385.

392 Guilford, T. C., and Biro, D. 2014. Route following and the pigeon's familiar area 393 map. J. Exp. Biol. 217: 169-179.

394 Guilford, T. C., and Taylor, G. K. 2014. The sun compass revisited. Anim. Behav. 395 97: 135-143.

396 Holland, R. A. 2003. The role of visual landmarks in the avian familiar area map. J.

397 Exp. Biol. 206: 1773-1778.

398 Jacobs, L. F. 2012. From chemotaxis to the cognitive map: the function of

399 olfaction. Proc. Natl. Acad. Sci. USA 109: Suppl. 1, 10693-10700.

400 Jorge, P. E., Marques, P. A. M., and Phillips, J. B. 2010. Activational effects of 401 odours on avian navigation. Proc. Roy. Soc. B, 277: 45-49.

402 Papi, F. 1992. Animal homing. Chapman and Hall, London.

403 Pearce, J. M. 2008. Animal Learning and Cognition: an Introduction. Hove, NY:

404 Psychology Press.

405 Wallraff, H. G. 2005. Avian Navigation. Pigeon homing as a Paradigm. Berlin:

406 Springer.

407 Weary, D., T. C. Guilford and R. Weisman 1993. A product of discriminative

408 learning may lead to female preferences for elaborate males. Evolution, 47: 333-

409336.

410 Wiltschko, R., and Wiltschko, W. 1995. Magnetic orientation in animals.

411 Springer, Berlin Heidelberg, New York. 
Wiltschko, R., \& Wiltschko, W. 2009. Avian navigation. The Auk 126: 717-743. 